\title{
Existence of solutions for fractional Schrödinger equation with asymptotica- lly periodic terms
}

\author{
Da-Bin Wang*, Man Guo, Wen Guan \\ Department of Applied Mathematics, Lanzhou University of Technology, 730050 Lanzhou, People's Republic of China.
}

Communicated by K. Q. Lan

\begin{abstract}
In this paper, we investigate the following nonlinear fractional Schrödinger equation

$$
(-\Delta)^{s} u+V(x) u=f(x, u), \quad x \in \mathbb{R}^{N},
$$

where $s \in(0,1), N>2$ and $(-\Delta)^{s}$ is fractional Laplacian operator. We prove that the problem has a non-trivial solution under asymptotically periodic case of $\mathrm{V}$ and $\mathrm{f}$ at infinity. Moreover, the nonlinear term $\mathrm{f}$ does not satisfy any monotone condition and Ambrosetti-Rabinowitz condition. (C)2017 All rights reserved.
\end{abstract}

Keywords: Fractional Schrödinger equation, asymptotically periodic, variational method. 2010 MSC: 34J10, 35J20, 35J60.

\section{Introduction}

In this paper, we consider the following fractional Schrödinger equations

$$
(-\triangle)^{s} u+V(x) u=f(x, u), \quad x \in \mathbb{R}^{N}
$$

where $s \in(0,1), \mathbf{N}>2,(-\triangle)^{s}$ is the fractional Laplacian operator of order s. Problem (1.1) arises the following nonlinear field equation

$$
i \frac{\partial \Psi}{\partial t}=(-\Delta)^{s} \Psi+(1+E) \Psi-K(x)|\Psi|^{q-1} \Psi, \quad x \in \mathbb{R}^{N}, \quad t \in \mathbb{R}^{+} .
$$

The nonlinear field equation (1.2) reflects the stable diffusion process of Lévy particles in random field. Later, people found that this stable diffusion of Lévy process has also a very important application in the mechanical system, flame propagation, chemical reactions in the liquid and the anomalous diffusion of the physics in the plasma. For more detail, readers can refer to $[5,20,21,36]$ and the references therein.

\footnotetext{
*Corresponding author

Email addresses: wangdb96@163.com (Da-Bin Wang), guoman615@163.com (Man Guo), mathguanw@163.com (Wen Guan)
} doi:10.22436/jnsa.010.02.25 
The problem (1.1) involves the fractional Laplacian $(-\triangle)^{s}$, which is a nonlocal operator. After this question was raised, it immediately aroused the interest of mathematicians [3, 6-11, 14, 16-18, 22, 2830, 34, 35, 38-40]. Especially, in [17], Felmer et al. studied the existence of positive solutions for the nonlinear fractional Schrödinger equation

$$
\left\{\begin{array}{l}
(-\Delta)^{s} u+u=f(x, u), \quad x \in \mathbb{R}^{N}, \\
u>0 \text { in } \mathbb{R}^{N}, \quad \lim _{|x| \rightarrow \infty} u(x)=0 .
\end{array}\right.
$$

Moreover, the regularity, decay and symmetry properties of these solutions of problem (1.3) were considered.

In [29], Secchi considered the existence of radially solutions for the following fractional Schrödinger equation

$$
(-\Delta)^{s} u+V(x) u=g(u), \quad x \in \mathbb{R}^{N} .
$$

In case $g(u)=|u|^{q-1} u, \lim _{|x| \rightarrow \infty} V(x)=+\infty$, Cheng [11] considered the existence of bound state solution of problem (1.4).

Later, when $\lim _{|x| \rightarrow \infty} V(x)=+\infty$, Secchi [28] obtained ground state solution for problem (1.4) with general nonlinearity $f(x, u)$.

Recently, Zhang et al. [38] studied ground state solution to problem (1.1). In [38], in addition to $V, f$ satisfies the asymptotic periodic condition, the nonlinear term $f$ satisfies the monotone condition: $t \mapsto$ $\frac{f(x, t)}{|t|}$ increasing on $(-\infty, 0)$ and $(0, \infty)$.

On the other hand, when $s=1$ the fractional Schrödinger equation (1.1) becomes the standard Schrödinger equation

$$
-\triangle \mathrm{u}+\mathrm{V}(\mathrm{x}) \mathrm{u}=\mathrm{f}(\mathrm{x}, \mathrm{u}), \quad x \in \mathbb{R}^{\mathrm{N}} .
$$

The Schrödinger equation (1.5) has been widely investigated by many authors in the last decades, see [2, $4,12,13,15,19,25,26,37]$ and references therein.

Motivated by above results, in this paper we study non-trivial solution and ground state solution to problem (1.1) under asymptotically periodic case of $V$ and $f$ at infinity. In the context about asymptotic periodic, we refer the reader to $[1,12,23,24,31,32]$.

Let $\Gamma$ be the functions $h \in C\left(\mathbb{R}^{N}, \mathbb{R}\right) \cap L^{\infty}\left(\mathbb{R}^{N}, \mathbb{R}\right)$ such that for every $\varepsilon>0$, the $\operatorname{set}\left\{x \in \mathbb{R}^{N}:|h(x)| \geqslant \varepsilon\right\}$ has finite Lebesgue measure. To state our main results, we assume that:

(V) There exist a constant $a_{0}>0$ and $V_{0} \in C\left(\mathbb{R}^{N}, \mathbb{R}\right), 1$-periodic in $x_{i}, 1 \leqslant i \leqslant N$, such that $V_{0}-V \in \Gamma$ and

$$
0<a_{0} \leqslant V(x) \leqslant V_{0}(x) .
$$

$\left(f_{1}\right)$ Set $F(x, t)=\int_{0}^{t} f(x, s) d s, F(x, t) \geqslant 0$ for all $(x, t) \in\left(\mathbb{R}^{N}, \mathbb{R}\right)$ and $f(x, t)=o(t)$ as $t \rightarrow 0$ uniformly in $x \in \mathbb{R}^{N}$.

$\left(\mathrm{f}_{2}\right)$ There exists a function $\mathrm{g} \in \mathrm{C}\left(\mathbb{R} /\{0\}, \mathbb{R}^{+}\right)$such that

$$
\hat{F}(x, t)=\frac{1}{2} f(x, t) t-F(x, t) \geqslant g(t) t^{2},
$$

for all $(x, t) \in\left(\mathbb{R}^{N}, \mathbb{R}\right)$.

$\left(f_{3}\right)$ There exist $a_{1}>0, R_{1}>0$ and $\tau>\frac{N}{2}$ such that

$$
|f(x, t)|^{\tau} \leqslant a_{1}|t|^{\tau} \hat{F}(x, t),
$$

for all $(x, t) \in\left(\mathbb{R}^{N}, \mathbb{R}\right)$ with $|t|>R_{1}$.

$\left(f_{4}\right) \lim _{|t| \rightarrow \infty} \frac{F(x, t)}{|t|^{2}}=+\infty$ uniformly in $x \in \mathbb{R}^{N}$. 
$\left(f_{5}\right)$ There exist $q \in\left(2,2_{s}^{*}\right)$, where $2_{s}^{*}=\frac{2 N}{N-2 s}$ and $h \in \Gamma, f_{0} \in C\left(\mathbb{R}^{N} \times \mathbb{R}, \mathbb{R}\right), 1$-periodic in $x_{i}, 1 \leqslant i \leqslant N$ such that

(i) $F(x, t) \geqslant F_{0}(x, t)=\int_{0}^{t} f_{0}(x, s) d s$, for all $(x, t) \in\left(\mathbb{R}^{N}, \mathbb{R}\right)$;

(ii) $\left|f(x, t)-f_{0}(x, t)\right| \leqslant h(x)|t|^{q-1}$, for all $(x, t) \in\left(\mathbb{R}^{N}, \mathbb{R}\right)$;

(iii) $\mathrm{t} \mapsto \frac{\mathrm{f}_{0}(\mathrm{x}, \mathrm{t})}{|\mathrm{t}|}$ is increasing in $(-\infty, 0)$ and $(0,+\infty)$.

Main results of this paper are as follows:

Theorem 1.1. Assume (V), $\left(\mathrm{f}_{1}\right)-\left(\mathrm{f}_{5}\right)$ are satisfied, then problem (1.1) has at least one non-trivial solution.

We also consider this condition:

$\left(f_{2}\right)^{\star} \hat{F}(x, t)>0$ for all $t \neq 0$.

Theorem 1.2. Suppose that $\mathrm{V}(\mathrm{x})$ and $\mathrm{f}(\mathrm{x}, \mathrm{t})$ are 1-periodic in $\mathrm{x}_{i}, 1 \leqslant i \leqslant \mathrm{~N}$ and $\mathrm{V}(\mathrm{x}) \geqslant \mathrm{a}_{0}>0$, for all $\mathrm{x} \in \mathbb{R}^{\mathrm{N}}$. If $\mathrm{f}$ satisfies $\left(\mathrm{f}_{1}\right),\left(\mathrm{f}_{3}\right),\left(\mathrm{f}_{4}\right)$ and $\left(\mathrm{f}_{2}\right)^{\star}$, then problem (1.1) has a ground-state solution.

Remark 1.3.

(1) In this paper, the condition $(\mathrm{V})$ and $\left(\mathrm{f}_{5}\right)$ mean asymptotically periodic case of $\mathrm{V}$ and $\mathrm{f}$ at infinity. This condition was introduced by Lins and Silva [24] in the study of a Schrödinger equation.

(2) In this paper, the condition $\left(f_{3}\right)$ is weaker than Ambrosetti-Rabinowitz condition. It is well-known that Ambrosetti-Rabinowitz condition has an important role in proof of bounded of Palais-Smale sequence. As far as we know, condition $\left(f_{3}\right)$ was introduced by Ding and Lee [15].

(3) In our paper, $f$ does not satisfy any monotone condition, that is, $\frac{f(x, t)}{t}$ is oscillatory and therefore the method of Nehari manifold [33] used in [38] is not applicable.

\section{Definitions and lemmas}

Let $s \in(0,1)$, the fractional Sobolev space $\mathrm{H}^{\mathrm{s}}\left(\mathbb{R}^{\mathrm{N}}\right)$ is defined by

$$
\mathrm{H}^{\mathrm{s}}\left(\mathbb{R}^{\mathrm{N}}\right)=\left\{\mathrm{u} \in \mathrm{L}^{2}\left(\mathbb{R}^{\mathrm{N}}\right): \frac{|\mathrm{u}(\mathrm{x})-\mathrm{u}(\mathrm{y})|}{|x-y|^{\frac{N}{2}+s}} \in \mathrm{L}^{2}\left(\mathbb{R}^{\mathrm{N}} \times \mathbb{R}^{\mathrm{N}}\right)\right\}
$$

and endowed with the natural norm

$$
\|u\|_{H^{s}\left(\mathbb{R}^{N}\right)}=\left(\int_{\mathbb{R}^{N}}|\mathfrak{u}|^{2} \mathrm{~d} x+\int_{\mathbb{R}^{N}} \int_{\mathbb{R}^{N}} \frac{|\mathfrak{u}(x)-u(y)|^{2}}{|x-y|^{N+2 s}} d x d y\right)^{\frac{1}{2}},
$$

here

$$
[u]_{H^{s}\left(\mathbb{R}^{N}\right)}=\left(\int_{\mathbb{R}^{N}} \int_{\mathbb{R}^{N}} \frac{|u(x)-u(y)|^{2}}{|x-y|^{N+2 s}} d x d y\right)^{\frac{1}{2}},
$$

is the so-called Gagliardo (semi) norm of $u$.

Using Fourier transform, the space $\mathrm{H}^{\mathrm{s}}\left(\mathbb{R}^{\mathrm{N}}\right)$ can also be defined by

$$
\mathrm{H}^{\mathrm{s}}\left(\mathbb{R}^{\mathrm{N}}\right)=\left\{u \in \mathrm{L}^{2}\left(\mathbb{R}^{\mathrm{N}}\right): \int_{\mathbb{R}^{\mathrm{N}}}\left(1+|\xi|^{2}\right)^{\mathrm{s}}|\mathrm{u}|^{2} \mathrm{~d} \xi<+\infty\right\},
$$

where $u$ denotes the Fourier transform of $u$.

Let $\ell$ be the Schwartz space of rapidly decreasing $C^{\infty}$ function on $\mathbb{R}^{N}, u \in \ell$, one has

$$
(-\triangle)^{s} u(x)=C(N, s) P . V . \int_{\mathbb{R}^{N}} \frac{u(x)-u(y)}{|x-y|^{N+2 s}} d y,
$$

the symbol P.V. stands for the Cauchy value and $C(N, s)$ is a constant depends only on the space dimen- 
sion $\mathrm{N}$ and the order $\mathrm{s}$.

From the results of $[7,14]$, we have

$$
(-\triangle)^{s} u={ }^{-1}\left(|\xi|^{2 s} u\right), \quad \forall \xi \in \mathbb{R}^{N} .
$$

Then, by Proposition 3.4 and Proposition 3.6 of $[7,14]$, we have

$$
[u]_{\mathrm{H}^{s}}^{2}=\frac{2}{\mathrm{C}(\mathrm{N}, \mathrm{s})} \int_{\mathbb{R}^{\mathrm{N}}}|\xi|^{2 s}|\mathrm{u}|^{2} \mathrm{~d} \xi=\frac{2}{\mathrm{C}(\mathrm{N}, \mathrm{s})}\left\|(-\triangle)^{\frac{\mathrm{s}}{2}} \mathrm{u}\right\|_{\mathrm{L}^{2}}^{2} \text {. }
$$

From the above fact, the norms on $\mathrm{H}^{\mathrm{s}}\left(\mathbb{R}^{\mathrm{N}}\right)$ defined below,

$$
\begin{gathered}
u \mapsto\left(\|u\|_{\mathrm{L}^{2}\left(\mathbb{R}^{N}\right)}^{2}+\int_{\mathbb{R}^{N}}|\xi|^{2 s}|u|^{2} d \xi\right)^{\frac{1}{2}}, \\
u \mapsto\left(\|u\|_{\mathrm{L}^{2}\left(\mathbb{R}^{N}\right)}^{2}+\left\|(-\triangle)^{\frac{s}{2}} u\right\|_{\mathrm{L}^{2}\left(\mathbb{R}^{N}\right)}^{2}\right)^{\frac{1}{2}}, \\
u \mapsto\|u\|_{H^{s}\left(\mathbb{R}^{N}\right)}
\end{gathered}
$$

are all equivalent.

In the sequel, we consider the Hilbert space $\mathrm{H}^{\mathrm{s}}\left(\mathbb{R}^{\mathrm{N}}\right)$ endowed with one of the following norms:

$$
\begin{aligned}
\|u\| & =\left(\int_{\mathbb{R}^{N}} \mathrm{~V}(\mathrm{x})|\mathrm{u}|^{2} \mathrm{~d} x+\int_{\mathbb{R}^{\mathrm{N}}}|\xi|^{2 \mathrm{~s}}|\mathrm{u}|^{2} \mathrm{~d} \xi\right)^{\frac{1}{2}}, \\
\|\mathrm{u}\|_{0} & =\left(\int_{\mathbb{R}^{N}} \mathrm{~V}_{0}(\mathrm{x})|\mathrm{u}|^{2} \mathrm{~d} x+\int_{\mathbb{R}^{\mathrm{N}}}|\xi|^{2 s}|\mathfrak{u}|^{2} \mathrm{~d} \xi\right)^{\frac{1}{2}},
\end{aligned}
$$

and the inner product induced by the first norm. In view of $(\mathrm{V})$, the norm $\|\cdot\|$ and $\|\cdot\|_{0}$ are equivalent to the standard norm in $\mathrm{H}^{\mathrm{s}}\left(\mathbb{R}^{\mathrm{N}}\right)$.

For the readers' convenience, we review the embedding results and Lions compactness lemma for the space $\mathrm{H}^{\mathrm{s}}\left(\mathbb{R}^{\mathrm{N}}\right)$.

Lemma $2.1([14,29])$. The embedding $\mathrm{H}^{\mathrm{s}}\left(\mathbb{R}^{\mathrm{N}}\right) \hookrightarrow \mathrm{L}^{\mathrm{q}}\left(\mathbb{R}^{\mathrm{N}}\right)$ is continuous for any $\mathrm{q} \in\left[2,2_{\mathrm{s}}^{*}\right]$. Moreover, The embedding $\mathrm{H}^{\mathrm{s}}\left(\mathbb{R}^{\mathrm{N}}\right) \hookrightarrow \mathrm{L}^{\mathrm{q}}\left(\mathbb{R}^{\mathrm{N}}\right)$ is locally compact whenever $\mathrm{q} \in\left[2,2_{\mathrm{s}}^{*}\right)$.

Lemma 2.2 ([28]). Assume $\mathrm{u}_{\mathrm{n}}$ is bounded in $\mathrm{H}^{\mathrm{s}}\left(\mathbb{R}^{\mathrm{N}}\right)$ and satisfies

$$
\lim _{n \rightarrow \infty} \sup _{y \in \mathbb{R}^{N}} \int_{B_{r}(y)}\left|u_{n}(x)\right|^{2} d x=0,
$$

for some $\mathrm{r}>0$. Then $\mathrm{u}_{\mathrm{n}} \rightarrow 0$ in $\mathrm{L}^{\mathrm{q}}\left(\mathbb{R}^{\mathrm{N}}\right)$ for any $\mathrm{q} \in\left(2,2_{\mathrm{s}}^{*}\right)$.

Lemma 2.3. Suppose that $\mathrm{f}$ satisfies $\left(\mathrm{f}_{1}\right),\left(\mathrm{f}_{3}\right)$ and (ii) of $\left(\mathrm{f}_{5}\right)$. Then for any given $\varepsilon>0$, there exist $\mathrm{C}_{\varepsilon}>0$ and $p \in\left(2,2_{s}^{*}\right)$ such that

$$
|\mathbf{f}(x, t)| \leqslant \varepsilon|t|+C_{\varepsilon}|t|^{p-1},|F(x, t)| \leqslant \varepsilon|t|^{2}+C_{\varepsilon}|t|^{p}, \text { for all }(x, t) \in\left(\mathbb{R}^{N}, \mathbb{R}\right) .
$$

Proof. Since the proof is easy, so we omitted here.

We say $u \in H^{s}\left(\mathbb{R}^{N}\right)$ is a weak solution of (1.1), if

$$
\int_{\mathbb{R}^{N}}|\xi|^{2 s} u v d \xi+\int_{\mathbb{R}^{N}} v(x) u v d x=\int_{\mathbb{R}^{N}} f(x, u) v d x,
$$


for all $v \in \mathrm{H}^{\mathrm{s}}\left(\mathbb{R}^{\mathrm{N}}\right)$.

The energy functional $\mathrm{I}: \mathrm{H}^{\mathrm{s}}\left(\mathbb{R}^{\mathrm{N}}\right) \rightarrow \mathbb{R}$ corresponding to problem (1.1) is defined by

$$
\mathrm{I}(\mathrm{u})=\frac{1}{2} \int_{\mathbb{R}^{N}}|\xi|^{2 s}|u|^{2} \mathrm{~d} \xi+\frac{1}{2} \int_{\mathbb{R}^{N}} \mathrm{~V}(\mathrm{x}) \mathrm{u}^{2} \mathrm{~d} x-\int_{\mathbb{R}^{N}} \mathrm{~F}(\mathrm{x}, \mathrm{u}) \mathrm{d} x .
$$

In fact, $\mathrm{I}(\mathrm{u})=\frac{1}{2}\|\mathrm{u}\|^{2}-\int_{\mathbb{R}^{\mathrm{N}}} \mathrm{F}(\mathrm{x}, \mathrm{u}) \mathrm{dx}$. In view of Lemma 2.3, the functional I is well-defined. Furthermore, under our condition, $I \in C^{1}\left(H^{s}\left(\mathbb{R}^{N}\right)\right)$ and its critical points are solutions of problem (1.1).

Let $u_{n} \subset H^{s}\left(\mathbb{R}^{N}\right)$, we say $u_{n}$ is a Cerami sequence for the functional I at level $c \in \mathbb{R}$, if

$$
\mathrm{I}\left(\mathrm{u}_{\mathrm{n}}\right) \rightarrow \mathrm{c},\left(1+\left\|\mathrm{u}_{\mathrm{n}}\right\|\right) \mathrm{I}^{\prime}\left(\mathrm{u}_{\mathrm{n}}\right) \rightarrow 0, \quad \mathrm{n} \rightarrow \infty .
$$

Theorem 2.4 ([27]). Let $\mathrm{E}$ be a real Banach space. Assume $\mathrm{I} \in \mathrm{C}^{\prime}(\mathrm{E}, \mathbb{R})$ satisfies $\mathrm{I}(0)=0$ and

$\left(\mathrm{I}_{1}\right)$ there exist $\rho, \alpha>0$ such that $\mathrm{I}(\mathrm{u}) \geqslant \alpha>0$, for all $\|\mathrm{u}\|=\rho$;

$\left(\mathrm{I}_{2}\right)$ there exists $\mathrm{e} \in \mathrm{E}$ with $\|\mathrm{e}\|>\rho$ such that $\mathrm{I}(\mathrm{e}) \leqslant 0$.

Then I possesses a Cerami sequence at level

$$
c=\inf _{\Theta} \max _{t \in[0,1]} I(\gamma(t))
$$

where

$$
\Theta=\{\gamma \in \mathrm{C}([0,1], \mathrm{E}): \gamma(0)=0,\|\gamma(1)\|>\rho, \mathrm{I}(\gamma(1)) \leqslant 0\} .
$$

Theorem 2.5 (local mountain pass theorem [24]). Let $\mathrm{E}$ be a real Banach space. Assume $\mathrm{I} \in \mathrm{C}^{\prime}(\mathrm{E}, \mathbb{R})$ satisfies $\mathrm{I}(0)=0,\left(\mathrm{I}_{1}\right)$ and $\left(\mathrm{I}_{2}\right)$. If there exists $\gamma_{0} \in \Theta, \Theta$ defined as in Theorem 2.4 such that

$$
\mathrm{c}=\max _{\mathrm{t} \in[0,1]} \mathrm{I}\left(\gamma_{0}(\mathrm{t})\right)>0,
$$

then I possesses a non-trivial critical point $u \in \gamma_{0}([0,1])$ at the level $\mathrm{c}$.

Lemma 2.6. Suppose that $\mathrm{f}$ satisfies $\left(\mathrm{f}_{1}\right),\left(\mathrm{f}_{3}\right),\left(\mathrm{f}_{4}\right)$ and (ii) of $\left(\mathrm{f}_{5}\right)$. Then $\mathrm{I}$ satisfies $\left(\mathrm{I}_{1}\right)$ and $\left(\mathrm{I}_{2}\right)$.

Proof. By Lemma 2.1 and Lemma 2.3, there exist $C>0, C_{1}>0$ such that

$$
\int_{\mathbb{R}^{N}} F(x, u) d x \leqslant \varepsilon|u|_{2}^{2}+C_{\varepsilon}|u|_{p}^{p} \leqslant \varepsilon C_{1}\|u\|^{2}+C\|u\|^{p} .
$$

Hence, we have

$$
\mathrm{I}(\mathrm{u})=\frac{1}{2}\|\mathrm{u}\|^{2}-\mathrm{C}_{1} \varepsilon\|\mathrm{u}\|^{2}-\mathrm{C}\|\mathrm{u}\|^{\mathrm{p}}=\left(\frac{1}{2}-\mathrm{C}_{1} \varepsilon\right)\|\mathrm{u}\|^{2}-\mathrm{C}\|\mathrm{u}\|^{\mathrm{p}} .
$$

Since $p>2$, we have

$$
\mathrm{I}(\mathrm{u}) \geqslant\left(\frac{1}{2}-\mathrm{C}_{1} \varepsilon\right)\|\mathrm{u}\|^{2}+\mathrm{o}\left(\|\mathrm{u}\|^{2}\right) \geqslant \alpha>0,
$$

for $\|u\|=\rho$ small enough. This proves $\left(\mathrm{I}_{1}\right)$.

To prove that there exists $e \in H^{s}\left(\mathbb{R}^{N}\right)$ such that $\mathrm{I}(e)<0$ let us choose $v \in \mathrm{H}^{\mathrm{s}}\left(\mathbb{R}^{\mathrm{N}}\right)$ such that $v(\mathrm{x}) \geqslant 0$ in $\mathbb{R}^{N}$ and $\|v\|=1$.

Let $\eta=\frac{2}{\int_{\mathbb{R}^{N}} v^{2} \mathrm{~d} x}$, by $\left(\mathrm{f}_{4}\right)$, there exists $\delta>0$ such that

$$
F(x, t) \geqslant \eta t^{2}, \quad \text { for }|t| \geqslant \delta .
$$

Let $R>0$, denote $\Omega_{R}=\left\{x \in \mathbb{R}^{N}: v(x) \geqslant \frac{\delta}{R}\right\}$, we have

$$
\int_{\mathbb{R}^{N}} F(x, R v) d x \geqslant \int_{\Omega_{R}} F(x, R v) d x \geqslant \eta R^{2} \int_{\Omega_{R}} v^{2} d x .
$$


Since $v \geqslant 0$, there exists $R_{0}>0$ such that for any $R \geqslant R_{0}$ we have that

$$
\int_{\Omega_{R}} v^{2} \mathrm{~d} x \geqslant \frac{1}{2} \int_{\mathbb{R}^{N}} v^{2} \mathrm{~d} x
$$

Then

$$
\int_{\mathbb{R}^{N}} F(x, R v) d x \geqslant \frac{\eta R^{2}}{2} \int_{\mathbb{R}^{N}} v^{2} d x
$$

From the above fact, one has that

$$
\mathrm{I}(\mathrm{R} v) \leqslant \frac{1}{2} \mathrm{R}^{2}\|v\|^{2}-\mathrm{R}^{2}<0,
$$

for any $\mathrm{R}>\mathrm{R}_{0}$. So, we choose $e=\mathrm{R} v$ and $\mathrm{I}(e)<0$.

The following lemma is a revised version of the corresponding lemma in [12], which we sketch here for the reader's convenience.

Lemma 2.7. Suppose that $\mathrm{f}$ satisfies $\left(\mathrm{f}_{1}\right)-\left(\mathrm{f}_{4}\right)$ and part (ii) of $\left(\mathrm{f}_{5}\right)$. Then any Cerami sequence for $\mathrm{I}$ is bounded.

Proof. Let $u_{n} \subset H^{s}\left(\mathbb{R}^{N}\right)$ such that

$$
\mathrm{I}\left(\mathrm{u}_{\mathrm{n}}\right) \rightarrow \mathrm{c}, \quad\left(1+\left\|\mathrm{u}_{\mathrm{n}}\right\|\right) \mathrm{I}^{\prime}\left(\mathrm{u}_{\mathrm{n}}\right) \rightarrow 0 .
$$

We have

$$
c+o_{n}(1)=I\left(u_{n}\right)-\frac{1}{2} I^{\prime}\left(u_{n}\right) u_{n}=\int_{\mathbb{R}^{N}} \hat{F}\left(x, u_{n}\right) d x .
$$

Suppose by contradiction that for some subsequences still denote $u_{n}$, we have that $\left\|u_{n}\right\| \rightarrow+\infty$.

Let $v_{n}=\frac{u_{n}}{\left\|u_{n}\right\|}$, one has

$$
o_{n}(1)=\frac{I^{\prime}\left(u_{n}\right) u_{n}}{\left\|u_{n}\right\|^{2}}=1-\int_{\mathbb{R}^{N}} \frac{f\left(x, u_{n}\right) v_{n}}{\left\|u_{n}\right\|} d x
$$

So,

$$
\lim _{n \rightarrow+\infty} \int_{\mathbb{R}^{N}} \frac{f\left(x, u_{n}\right) v_{n}}{\left\|u_{n}\right\|} d x=1
$$

Let

$$
G(r)=\inf \left\{\hat{F}(x, t): x \in \mathbb{R}^{N},|t| \geqslant r\right\},
$$

where $r \geqslant 0$.

By $\left(f_{2}\right)$ and $\left(f_{3}\right)$, for any $|t|>R_{1}$, we have

$$
a_{1} \hat{F}(x, t) \geqslant\left(\frac{f(x, t)}{t}\right)^{r} \geqslant\left(\frac{2 F(x, t)}{t^{2}}\right)^{\tau}
$$

According to $\left(f_{4}\right), \hat{F}(x, t) \rightarrow \infty, t \rightarrow \infty$ uniformly in $x \in \mathbb{R}^{\mathbb{N}}$. So, it is easy to see that $G(r)>0$ for all $r>0$ and $\mathrm{G}(\mathrm{r}) \rightarrow \infty$ as $\mathrm{r} \rightarrow \infty$.

Let $0<\mathrm{a}<\mathrm{b}$, denote

$$
\Omega_{\mathrm{n}}(\mathrm{a}, \mathrm{b})=\left\{x \in \mathbb{R}^{\mathrm{N}}: \mathrm{a} \leqslant\left|\mathrm{u}_{\mathrm{n}}(x)\right|<\mathrm{b}\right\} .
$$

Let $0 \leqslant \mathrm{a}<\mathrm{b}$, denote

$$
c_{a}^{b}=\inf \left\{\frac{\hat{F}(x, t)}{t^{2}}: x \in \mathbb{R}^{N}, a \leqslant|t| \leqslant b\right\}
$$

In view of $\left(f_{2}\right), c_{a}^{b}>0$.

Above all, we have that

$$
c+o_{n}(1)=\int_{\Omega_{n}(0, a)} \hat{F}\left(x, u_{n}\right) d x+\int_{\Omega_{n}(a, b)} \hat{F}\left(x, u_{n}\right) d x+\int_{\Omega_{n}(b, \infty)} \hat{F}\left(x, u_{n}\right) d x
$$




$$
\geqslant \int_{\Omega_{n}(0, a)} \hat{F}\left(x, u_{n}\right) d x+c_{a}^{b} \int_{\Omega_{n}(a, b)} u_{n}^{2} d x+G(b)\left|\Omega_{n}(b, \infty)\right| .
$$

Therefore, there exists $C_{1}>0$ such that

$$
\max \left\{\int_{\Omega_{n}(0, a)} \hat{F}\left(x, u_{n}\right) d x, \quad c_{a}^{b} \int_{\Omega_{n}(a, b)} u_{n}^{2} d x, G(b)\left|\Omega_{n}(b, \infty)\right|\right\} \leqslant C_{1} .
$$

Since the fact that $\mathrm{G}(\mathrm{b}) \rightarrow+\infty$ as $\mathrm{b} \rightarrow+\infty$, one gets $\lim _{\mathrm{b} \rightarrow+\infty}\left|\Omega_{\mathfrak{n}}(\mathrm{b}, \infty)\right|=0$.

Let $\mu \in\left[2,2_{s}^{*}\right)$, by Hölder's inequality and Lemma 2.1 , there exists $C_{2}>0$ such that

$$
\begin{aligned}
\int_{\Omega_{n}(b, \infty)}\left|v_{n}\right|^{\mu} \mathrm{d} x & \leqslant\left(\int_{\Omega_{\Omega_{n}(b, \infty)}}\left|v_{n}\right|^{2_{s}^{*}} \mathrm{~d} x\right)^{\mu / 2_{s}^{*}}\left|\Omega_{n}(b, \infty)\right|^{\left(2_{s}^{*}-\mu\right) / 2_{s}^{*}} \\
& \leqslant C_{2}\left\|v_{n}\right\|^{\mu}\left|\Omega_{n}(b, \infty)\right|^{\left(2_{s}^{*}-\mu\right) / 2_{s}^{*}}=C_{2}\left|\Omega_{n}(b, \infty)\right|^{\left(2_{s}^{*}-\mu\right) / 2_{s}^{*}} .
\end{aligned}
$$

Since $\mu<2_{s}^{*}$, we conclude that

$$
\lim _{\mathrm{b} \rightarrow+\infty} \int_{\Omega_{\mathrm{n}}(\mathrm{b}, \infty)}\left|v_{\mathrm{n}}\right|^{\mu} \mathrm{d} \mathrm{x}=0 .
$$

Let $\varepsilon \in\left(0, \frac{1}{3}\right)$, by $\left(f_{1}\right)$ there exists $a_{\varepsilon}>0$ such that

$$
|f(x, t)| \leqslant \frac{\varepsilon|t|}{C_{3}^{2}}, \text { for all }|t| \leqslant a_{\varepsilon},
$$

where $C_{3}>0$ be such that $|u|_{2} \leqslant C_{3}\|u\|$ for all $u \in H^{s}\left(\mathbb{R}^{N}\right)$. Hence,

$$
\int_{\Omega_{n}\left(0, a_{\varepsilon}\right)} \frac{f\left(x, u_{n}\right) v_{n}}{\left\|u_{n}\right\|} d x \leqslant \frac{\varepsilon}{C_{3}^{2}} \int_{\Omega_{n}\left(0, a_{\varepsilon}\right)} v_{n}^{2} d x \leqslant \varepsilon .
$$

Let $2 \tau^{\prime}=2 \tau /(\tau-1) \in\left(2,2_{s}^{*}\right)$, from $\left(f_{3}\right),(2.2)$ and Hölder's inequality one has

$$
\begin{aligned}
\int_{\Omega_{\mathfrak{n}}\left(b_{\varepsilon}, \infty\right)} \frac{f\left(x, u_{n}\right) v_{n}}{\left\|u_{n}\right\|} d x & \leqslant \int_{\Omega_{n}\left(b_{\varepsilon}, \infty\right)} \frac{f\left(x, u_{n}\right) v_{n}^{2}}{\left|u_{n}\right|} d x \\
& \leqslant\left(\int_{\Omega_{n}\left(b_{\varepsilon}, \infty\right)} \frac{\left|f\left(x, u_{n}\right)\right|^{\tau}}{\left|u_{n}\right|^{\tau}} d x\right)^{1 / \tau}\left(\int_{\Omega_{n}\left(b_{\varepsilon}, \infty\right)}\left|v_{n}\right|^{2 \tau^{\prime}} d x\right)^{1 / \tau^{\prime}} \\
& \leqslant a_{1}^{1 / \tau}\left(\int_{\Omega_{n}\left(b_{\varepsilon}, \infty\right)} \hat{\mathrm{F}}\left(x, u_{n}\right) d x\right)^{1 / \tau}\left(\int_{\Omega_{n}\left(b_{\varepsilon}, \infty\right)}\left|v_{n}\right|^{2 \tau^{\prime}} d x\right)^{1 / \tau^{\prime}} \\
& \leqslant C_{5}\left(\int_{\Omega_{n}\left(b_{\varepsilon}, \infty\right)}\left|v_{n}\right|^{2 \tau^{\prime}} d x\right)^{1 / \tau^{\prime}} .
\end{aligned}
$$

So by (2.3), for $b_{\varepsilon}>0$ sufficiently large we have

$$
\int_{\Omega_{n}\left(b_{\varepsilon}, \infty\right)} \frac{f\left(x, u_{n}\right) v_{n}}{\left\|u_{n}\right\|} d x<\varepsilon
$$

In view of (ii) of $\left(f_{5}\right)$ and $f_{0} \in C\left(\mathbb{R}^{N} \times \mathbb{R}, \mathbb{R}\right)$, there exists $C_{4}>0$ such that $\left|f\left(x, u_{n}\right)\right| \leqslant C_{4}\left|u_{n}\right|$, for every $x \in \Omega_{n}\left(a_{\varepsilon}, b_{\varepsilon}\right)$. From (2.1), there exists $n_{0}$ such that $n \geqslant n_{0}$ we have

$$
\int_{\Omega_{n}\left(a_{\varepsilon}, b_{\varepsilon}\right)} \frac{f\left(x, u_{n}\right) v_{n}}{\left\|u_{n}\right\|} d x \leqslant C_{4} \int_{\Omega_{n}\left(a_{\varepsilon}, b_{\varepsilon}\right)} v_{n}^{2} d x \leqslant \frac{C_{1} C_{4}}{c_{a}^{b}\left\|u_{n}\right\|^{2}}<\epsilon .
$$

Hence, according to (2.4), (2.5), (2.6) for $b_{\varepsilon}>0$ sufficiently large we have

$$
\int_{\mathbb{R}^{N}} \frac{f\left(x, u_{n}\right) v_{n}}{\left\|u_{n}\right\|} d x \leqslant 3 \varepsilon<1,
$$

which contradicts (2.1). Therefore $\left\{u_{n}\right\}$ is bounded in $H^{s}\left(\mathbb{R}^{N}\right)$. 
Remark 2.8. The above lemma still holds under the conditions of Theorem 1.2.

Lemma 2.9. Suppose that $\mathrm{f}$ satisfies $\left(\mathrm{f}_{1}\right)-\left(\mathrm{f}_{4}\right)$ and (ii) of $\left(\mathrm{f}_{5}\right)$. Let $\mathrm{u}_{\mathrm{n}} \subset \mathrm{H}^{\mathrm{s}}\left(\mathbb{R}^{\mathrm{N}}\right)$ be Cerami sequence for $\mathrm{I}$ at level $c>0$. If $u_{n} \rightarrow 0$ in $\mathrm{H}^{\mathrm{s}}\left(\mathbb{R}^{\mathrm{N}}\right)$, then there exist a sequence $\left\{\mathrm{y}_{\mathrm{n}}\right\} \subset \mathbb{R}^{\mathrm{N}}$ and $\mathrm{R}>0, \beta>0$ such that $\mathrm{y}_{\mathrm{n}} \rightarrow \infty$ and

$$
\lim _{n \rightarrow \infty} \sup \int_{B_{R}\left(y_{n}\right)}\left|u_{n}\right|^{2} \geqslant \beta>0 .
$$

Proof. Suppose by contradiction that the lemma is false. Then for any $\mathrm{R}>0$, we have that

$$
\lim _{n \rightarrow \infty} \sup \int_{B_{R}(y)}\left|u_{n}\right|^{2}=0,
$$

for all $R>0$. By Lemma 2.2, we have that $\left|u_{n}\right|_{L^{s}} \rightarrow 0$ for any $s \in\left(2,2_{s}^{*}\right)$.

By Lemma 2.3, we have

$$
\lim _{n \rightarrow \infty} \int_{\mathbb{R}^{N}} f\left(x, u_{n}\right) u_{n} d x \leqslant \lim _{n \rightarrow \infty}\left(\varepsilon \int_{\mathbb{R}^{N}}\left|u_{n}\right|^{2}+C_{\varepsilon} \int_{\mathbb{R}^{N}}\left|u_{n}\right|^{p}\right) .
$$

In view of the boundedness of $u_{n}$ in $L^{2}\left(\mathbb{R}^{N}\right)$ and $\varepsilon$ is arbitrary, we get that

$$
\int_{\mathbb{R}^{N}} f\left(x, u_{n}\right) u_{n} d x \rightarrow 0
$$

as $n \rightarrow \infty$.

Similarly, we have that $\int_{\mathbb{R}^{N}} F\left(x, u_{n}\right) d x \rightarrow 0$ as $n \rightarrow \infty$. So we get

$$
c+o_{n}(1)=I\left(u_{n}\right)-\frac{1}{2} I^{\prime}\left(u_{n}\right) u_{n}=\int_{\mathbb{R}^{N}}\left(\frac{1}{2} f\left(x, u_{n}\right) u_{n}-F\left(x, u_{n}\right)\right) d x .
$$

Therefore, $\mathrm{c}=0$ which contradicts with $\mathrm{c}>0$.

Lemma $2.10([31,38])$. Assume $\left\{u_{n}\right\} \subset \mathrm{H}^{s}\left(\mathbb{R}^{N}\right)$ satisfies $u_{n} \rightarrow 0$ and $\left\{\varphi_{n}\right\} \subset \mathrm{H}^{s}\left(\mathbb{R}^{N}\right)$ is bounded. Then

$$
\begin{gathered}
\int_{\mathbb{R}^{N}}\left[V(x)-V_{0}(x)\right] u_{n} \varphi_{n} d x \rightarrow 0, \\
\int_{\mathbb{R}^{N}}\left[f\left(x, u_{n}\right)-f_{0}\left(x, u_{n}\right)\right] \varphi_{n} d x \rightarrow 0 .
\end{gathered}
$$

Lemma 2.11. Assume $\mathrm{h} \in \Gamma$ and $\mu \in\left[2,2_{\mathrm{s}}^{*}\right]$. If $\mathrm{u}_{\mathrm{n}} \subset \mathrm{H}^{\mathrm{s}}\left(\mathbb{R}^{\mathrm{N}}\right)$ such that $\mathrm{u}_{\mathrm{n}} \rightarrow \mathrm{u}$ weakly in $\mathrm{H}^{\mathrm{s}}\left(\mathbb{R}^{\mathrm{N}}\right)$, then

$$
\lim _{n \rightarrow+\infty} \int_{\mathbb{R}^{N}} h\left|u_{n}\right|^{\mu} d x=\int_{\mathbb{R}^{N}} h|u|^{\mu} d x .
$$

Proof. Since the proof is similar to that of the results in [31], so we omitted here.

\section{Proof of theorems}

Let $\mathrm{I}_{0}: \mathrm{H}^{\mathrm{s}}\left(\mathbb{R}^{\mathrm{N}}\right) \rightarrow \mathbb{R}$ be the functional associated with the periodic problem, namely,

$$
\mathrm{I}_{0}(\mathrm{u})=\frac{1}{2} \int_{\mathbb{R}^{\mathrm{N}}}|\xi|^{2 \mathrm{~s}}|\mathrm{u}|^{2} \mathrm{~d} \xi+\frac{1}{2} \int_{\mathbb{R}^{\mathrm{N}}} \mathrm{v}_{0}(\mathrm{x}) \mathrm{u}^{2} \mathrm{~d} x-\int_{\mathbb{R}^{\mathrm{N}}} \mathrm{F}_{0}(\mathrm{x}, \mathrm{u}) \mathrm{d} \mathrm{x}
$$

Proof of Theorem 1.1. In view of Lemma 2.6 and Theorem 2.4, there exists a Cerami sequence $\left\{u_{n}\right\} \subset$ $\mathrm{H}^{\mathrm{s}}\left(\mathbb{R}^{\mathrm{N}}\right)$, i.e.,

$$
\mathrm{I}^{\prime}\left(\mathrm{u}_{\mathrm{n}}\right) \rightarrow \mathrm{c} \geqslant \alpha>0, \text { and }\left(1+\left\|\mathrm{u}_{\mathrm{n}}\right\|\right) \mathrm{I}^{\prime}\left(\mathrm{u}_{\mathrm{n}}\right) \rightarrow 0, \text { as } \mathrm{n} \rightarrow \infty .
$$

From Lemma 2.7, $\left\{u_{n}\right\}$ is bounded. Going if necessary to a subsequence, one assumes that $u_{n} \rightarrow u$ weakly in $\mathrm{H}^{\mathrm{s}}\left(\mathbb{R}^{\mathrm{N}}\right)$. 
To prove $I^{\prime}(u)=0$. In fact, it suffices to prove that $I^{\prime}(u) \varphi=0$ for all $\varphi \in C_{0}^{\infty}\left(\mathbb{R}^{N}\right)$, since $C_{0}^{\infty}\left(\mathbb{R}^{N}\right)$ is dense in $\mathrm{H}^{\mathrm{s}}\left(\mathbb{R}^{\mathrm{N}}\right)$. For all $\varphi \in \mathrm{C}_{0}^{\infty}\left(\mathbb{R}^{\mathrm{N}}\right)$, we have

$$
\begin{aligned}
I^{\prime}\left(u_{n}\right) \varphi-I^{\prime}(u) \varphi= & \int_{\mathbb{R}^{N}}|\xi|^{2 s} u_{n} \varphi d \xi+\int_{\mathbb{R}^{N}} V(x) u_{n} \varphi d x-\int_{\mathbb{R}^{N}} f\left(x, u_{n}\right) \varphi d x \\
& -\int_{\mathbb{R}^{N}}|\xi|^{2 s} u \varphi d \xi-\int_{\mathbb{R}^{N}} V(x) u \varphi d x+\int_{\mathbb{R}^{N}} f(x, u) \varphi d x \\
= & \left\langle u_{n}-u, \varphi\right\rangle-\int_{\mathbb{R}^{N}}\left(f\left(x, u_{n}\right)-f(x, u)\right) \varphi d x .
\end{aligned}
$$

Since $u_{n} \rightarrow u$ weakly in $H^{s}\left(\mathbb{R}^{N}\right)$ and Lemma 2.3, we have

$$
\mathrm{I}^{\prime}(\mathrm{u}) \varphi=\lim _{\mathrm{n} \rightarrow \infty} \mathrm{I}^{\prime}\left(\mathrm{u}_{\mathrm{n}}\right) \varphi=0,
$$

which implies that $\mathrm{I}^{\prime}(u)=0$.

If $u \neq 0$, the proof is finished.

If $u=0$, from Lemma 2.9, there exist a sequence $\left(y_{n}\right) \subset \mathbb{R}^{N}, R>0$ and $\beta>0$ such that $\left|y_{n}\right| \rightarrow \infty$ as $n \rightarrow \infty$ and

$$
\limsup _{n \rightarrow \infty} \int_{B_{R\left(y_{n}\right)}}\left|u_{n}\right|^{2} \geqslant \beta>0 .
$$

Let $\left\{y_{n}\right\} \subset \mathbb{Z}^{N}$ and $\tilde{u}_{n}(x)=u_{n}\left(x+y_{n}\right)$ and $\left\|\tilde{u}_{n}\right\|=\left\|u_{n}\right\|_{0}$. Going if necessary to a subsequence, from Lemma 2.1 we can assume that

$$
\begin{gathered}
\tilde{u}_{n} \rightarrow \tilde{u} \in H^{s}\left(\mathbb{R}^{N}\right), \\
\tilde{u}_{n} \rightarrow \tilde{u} \in L_{\text {loc }}^{q}\left(\mathbb{R}^{N}\right), \quad 2 \leqslant q<2_{s}^{*}, \\
\tilde{u}_{n} \rightarrow \tilde{u} \text { a.e in } \mathbb{R}^{N} .
\end{gathered}
$$

From (3.2), we have $\tilde{u} \neq 0$.

To prove $\mathrm{I}_{0}^{\prime}(\tilde{\mathrm{u}})=0$ :

For all $\varphi \in \mathrm{C}_{0}^{\infty}\left(\mathbb{R}^{N}\right)$, for each $n \in \mathbb{N}$, let $\varphi_{n}(x)=\varphi\left(x-y_{n}\right)$, we have

$$
\mathrm{I}_{0}^{\prime}(\tilde{\mathrm{u}}) \varphi=\mathrm{I}_{0}^{\prime}\left(\tilde{u}_{\mathrm{n}}\right) \varphi+\mathrm{o}_{\mathfrak{n}}(1)=\mathrm{I}_{0}^{\prime}\left(\mathrm{u}_{\mathrm{n}}\right) \varphi_{\mathrm{n}}+\mathrm{o}_{\mathfrak{n}}(1) .
$$

On the other hand, by Lemma 2.10 one has that

$$
\begin{aligned}
& I_{0}^{\prime}\left(u_{n}\right) \varphi_{n}=I^{\prime}\left(u_{n}\right) \varphi_{n}+\int_{\mathbb{R}^{N}}\left[V_{0}(x)-V(x)\right] u_{n} \varphi_{n} d x-\int_{\mathbb{R}^{N}}\left[f_{0}\left(x, u_{n}\right)-f(x, u)\right] \varphi_{n} d x \\
& =\mathrm{I}^{\prime}\left(\mathrm{u}_{\mathrm{n}}\right) \varphi_{\mathrm{n}}+\mathrm{o}_{\mathrm{n}}(1) \text {. }
\end{aligned}
$$

From (3.1), we have at $\mathrm{I}_{0}^{\prime}(\tilde{\mathrm{u}})=0$. From (ii) of $\left(\mathrm{f}_{5}\right)$, one has that

$$
\left|\hat{\mathrm{F}}(x, t)-\hat{\mathrm{F}}_{0}(x, t)\right| \leqslant\left(\frac{1}{2}+\frac{1}{\mathrm{q}}\right) \mathrm{h}(x)|\mathrm{t}|^{\mathrm{q}} .
$$

According to $u_{n} \rightarrow 0$ weakly in $H^{s}\left(\mathbb{R}^{N}\right)$ and Lemma 2.11 we have that

$$
\lim _{n \rightarrow \infty} \int_{\mathbb{R}^{N}} \hat{F}\left(x, u_{n}\right) d x=\lim _{n \rightarrow \infty} \int_{\mathbb{R}^{N}} \hat{F}_{0}\left(x, u_{n}\right) d x=\lim _{n \rightarrow \infty} \int_{\mathbb{R}^{N}} \hat{F}_{0}\left(x, \tilde{u}_{n}\right) d x \geqslant \int_{\mathbb{R}^{N}} \hat{F}_{0}(x, \tilde{u}) d x .
$$

By (3.1), we have

$$
c=\lim _{n \rightarrow \infty}\left[I\left(u_{n}\right)-\frac{1}{2} I^{\prime}\left(u_{n}\right) u_{n}\right]=\liminf _{n \rightarrow \infty} \int_{\mathbb{R}^{N}} \hat{F}\left(x, u_{n}\right) d x \geqslant \int_{\mathbb{R}^{N}} \hat{F}_{0}(x, \tilde{u}) d x
$$




$$
\begin{aligned}
& =\mathrm{I}_{0}(\tilde{\mathrm{u}})-\frac{1}{2} \mathrm{I}_{0}^{\prime}(\tilde{\mathrm{u}}) \tilde{\mathrm{u}} \\
& =\mathrm{I}_{0}(\tilde{\mathrm{u}}) .
\end{aligned}
$$

That is $\mathrm{I}_{0}(\tilde{\mathrm{u}}) \leqslant \mathrm{c}$.

Next we prove $\max _{t \geqslant 0} I_{0}(t \tilde{u})=I_{0}(\tilde{u})$. Let

$$
\chi(t)=I_{0}(t \tilde{u})=\frac{t^{2}}{2}\|\tilde{u}\|_{0}^{2}-\int_{\mathbb{R}^{N}} F_{0}(x, t \tilde{u}) d x,
$$

then

$$
\begin{aligned}
x^{\prime}(t) & =t\|\tilde{u}\|_{0}^{2}-\int_{\mathbb{R}^{N}} f_{0}(x, t \tilde{u}) \tilde{u} d x \\
& =t\left(\|\tilde{u}\|_{0}^{2}-\int_{\mathbb{R}^{N}} \frac{f_{0}(x, t \tilde{u}) \tilde{u}}{t}\right) d x \\
& =t A(t) .
\end{aligned}
$$

Since $I_{0}^{\prime}(\tilde{u})=0, A(1)=0$ from (iii) of $\left(f_{5}\right), A$ is nonincreasing in $(0, \infty)$. Then $A(t)>0$ when $t \in$ $(0,1)$ and $A(t)<0$ when $t \in(1, \infty)$. Therefore

$$
\chi^{\prime}(t)>0 \text {, when } t \in(0,1) \text { and } \chi^{\prime}(t)<0 \text {, when } t \in(1, \infty) .
$$

Above all, we have that $\max _{t \geqslant 0} I_{0}(t \tilde{u})=I_{0}(\tilde{u})$. Hence, by the definition of $c,(V)$ and part (i) of $\left(f_{5}\right)$, we have that

$$
c \leqslant \max _{t \geqslant 0} I(t \tilde{u}) \leqslant \max _{t \geqslant 0} I_{0}(t \tilde{u})=I_{0}(\tilde{u}) \leqslant c .
$$

By Theorem 2.5, we obtain that I possesses a critical point at level $c>0$. So the proof is finished.

Proof of Theorem 1.2. It is easy to see that Lemmas 2.3, 2.6 and 2.7 are all held by using the conditions of Theorem 1.2. From Lemma 2.6 and Theorem 2.4, there exists Cerami sequence $\left\{u_{n}\right\} \subset H^{s}\left(\mathbb{R}^{N}\right)$, i.e.,

$$
\mathrm{I}_{0}\left(\mathrm{u}_{\mathrm{n}}\right) \rightarrow \mathrm{c}_{0}, \text { and }\left(1+\left\|\mathrm{u}_{\mathrm{n}}\right\|_{0}\right) \mathrm{I}_{0}^{\prime}\left(\mathrm{u}_{\mathrm{n}}\right) \rightarrow 0, \text { as } \mathrm{n} \rightarrow+\infty,
$$

where $c_{0}$ is the mountain pass level of $\mathrm{I}_{0}$.

By Lemma 2.7, we conclude that $u_{n} \rightarrow u$ weakly in $H^{s}\left(\mathbb{R}^{N}\right)$. Similar to proof of Theorem 1.1, we have $\mathrm{I}_{0}^{\prime}(\mathrm{u})=0$.

We only need to consider the case in which $u=0$. By Lemma 2.9, there are a sequence $\left(y_{n}\right) \subset \mathbb{Z}^{N}$, $\mathrm{R}>0$ and $\beta>0$ such that $\left|\mathrm{y}_{\mathrm{n}}\right| \rightarrow \infty$ as $\mathrm{n} \rightarrow \infty$ and

$$
\limsup _{n \rightarrow \infty} \int_{B_{R\left(y_{n}\right)}}\left|u_{n}\right|^{2} \geqslant \beta>0 .
$$

Let $\tilde{u}_{n}(x)=u_{n}\left(x+y_{n}\right)$, then $\left\|\tilde{u}_{n}\right\|_{0}=\left\|u_{n}\right\|_{0}$. Up to a subsequence, we have

$$
\begin{gathered}
\tilde{u}_{n} \rightarrow \tilde{u} \text { weakly in } H^{s}\left(\mathbb{R}^{N}\right), \\
\tilde{u}_{n} \rightarrow \tilde{u} \text { in } L_{\text {loc }}^{2}\left(\mathbb{R}^{N}\right), \\
\tilde{u}_{n}(x) \rightarrow \tilde{u} \text { almost every where in } \mathbb{R}^{N} .
\end{gathered}
$$

By (3.3), $\tilde{u} \neq 0$. Similar to proof of Theorem 1.1, we get $I_{0}^{\prime}(\tilde{u})=0$.

So $m=\inf \left\{I_{0}(u): u \in H^{s}\left(\mathbb{R}^{N}\right), I^{\prime}(u)=0\right\}>0$ is well-defined. Next, we prove $m$ is achieved. Indeed, let $\left\{u_{n}\right\} \subset H^{s}\left(\mathbb{R}^{N}\right)$ be a minimizing sequence for $m$, i.e.,

$$
\mathrm{I}_{0}\left(\mathrm{u}_{\mathrm{n}}\right) \rightarrow \mathrm{m}, \quad \mathrm{I}_{0}^{\prime}\left(\mathrm{u}_{\mathrm{n}}\right)=0 \text { and } \mathrm{u}_{\mathrm{n}} \neq 0
$$


Obviously, $\left\{u_{n}\right\}$ is a Cerami sequence for $I_{0}$. So, from Lemma 2.7, $\left\{u_{n}\right\}$ is bounded. Moreover, from $I_{0}^{\prime}\left(u_{n}\right) u_{n}=0$ and Lemma 2.3, there exists $\alpha>0$ such that $\left\|u_{n}\right\|_{0} \geqslant \alpha$. Thus, arguing as in the preceding paragraph, we obtain a translated subsequence $\left\{\tilde{u}_{n}\right\}$, which has a non-zero weak limit $\mathfrak{u}_{0}$ such that $\mathrm{I}_{0}^{\prime}\left(\mathrm{u}_{0}\right)=0$ and $\tilde{u}_{n}(x) \rightarrow u_{0}(x)$ a.e. in $\mathbb{R}^{\mathrm{N}}$. By Fatou's lemma,

$$
m=\lim _{n \rightarrow \infty} I_{0}\left(u_{n}\right)=\lim _{n \rightarrow \infty} I_{0}\left(\tilde{u}_{n}\right)=\liminf _{n \rightarrow \infty} \int_{\mathbb{R}^{N}} \hat{F}_{0}\left(x, \tilde{u}_{n}\right) d x \geqslant \int_{\mathbb{R}^{N}} \hat{F}_{0}\left(x, u_{0}\right) d x=I_{0}\left(u_{0}\right) .
$$

Hence, $I_{0}\left(u_{0}\right)=m$, that is $u_{0} \neq 0$ is a ground-state solution of problem (1.1).

\section{Acknowledgment}

Research was supported by NSFC (11561043). The authors express their sincere thanks to the reviewers and editor for the useful suggestions to improve the paper.

\section{References}

[1] C. O. Alves, M. A. S. Souto, S. H. M. Soares, Schrödinger-Poisson equations without Ambrosetti-Rabinowitz condition, J. Math. Anal. Appl., 377 (2011), 584-592. 1

[2] A. Ambrosetti, M. Badiale, S. Cingolani, Semiclassical states of nonlinear Schrödinger equations, Arch. Rational Mech. Anal., 140 (1997), 285-300. 1

[3] B. Barrios, E. Colorado, A. de Pablo, U. Sánchez, On some critical problems for the fractional Laplacian operator, J. Differential Equations, 252 (2011), 6133-6162. 1

[4] H. Berestycki, P.-L. Lions, Nonlinear scalar field equations, II, Existence of infinitely many solutions, Arch. Rational Mech. Anal., 82 (1983), 347-375. 1

[5] J. Bertoin, Léry Processes, Cambridge Tracts in Mathematics, Cambridge University Press, Cambridge, (1996). 1

[6] G. M. Bisci, V. D. Rădulescu, Ground state solutions of scalar field fractional Schrödinger equations, Calc. Var. Partial Differential Equations, 54 (2015), 2985-3008. 1

[7] G. M. Bisci, V. D. Rădulescu, R. Servadei, Variational methods for nonlocal fractional problems, With a foreword by Jean Mawhin, Encyclopedia of Mathematics and its Applications, Cambridge University Press, Cambridge, (2016). 2

[8] X. Cabré, J.-G. Tan, Positive solutions of nonlinear problems involving the square root of the Laplacian, Adv. Math., 224 (2010), 2052-2093.

[9] L. Caffarelli, L. Silvestre, An extension problem related to the fractional Laplacian, Comm. Partial Differential Equations, 32 (2007), 1245-1260.

[10] X. Chang, Z.-Q. Wang, Ground state of scalar field equations involving a fractional Laplacian with general nonlinearity, Nonlinearity, 26 (2013), 479-494.

[11] M. Cheng, Bound state for the fractional Schrödinger equation with unbounded potential, J. Math. Phys., 54 (2012), 7 pages. 1, 1

[12] R. de Marchi, Schrödinger equations with asymptotically periodic terms, Proc. Roy. Soc. Edinburgh Sect. A, 145 (2015), 745-757. 1, 2

[13] M. del Pino, P. Felmer, Semi-classical states of nonlinear Schrdinger equations: a variational reduction method, Math. Ann., 324 (2002), 1-32. 1

[14] E. Di Nezza, G. Palatucci, E. Valdinoci, Hitchhiker's guide to the fractional Sobolev spaces, Bull. Sci. Math., 136 (2012), 521-573. 1, 2, 2.1

[15] Y.-H. Ding, F.-H. Lin, Solutions of perturbed Schrödinger equations with critical nonlinearity, Calc. Var. Partial Differential Equations, 30 (2007), 231-249. 1, 1.3

[16] S. Dipierro, G. Palatucci, E. Valdinoci, Existence and symmetry results for a Schrödinger type problem involving the fractional Laplacian, Matematiche (Catania), 68 (2013), 201-216. 1

[17] P. Felmer, A. Quaas, J.-G. Tan, Positive solutions of the nonlinear Schrödinger equation with the fractional Laplacian, Proc. Roy. Soc. Edinburgh Sect. A, 142 (2012), 1237-1262. 1

[18] J. Giacomoni, P. K. Mishra, K. Sreenadh, Fractional elliptic equations with critical exponential nonlinearity, Adv. Nonlinear Anal., 5 (2016), 57-74. 1

[19] L. Jeanjean, K. Tanaka, Singularly perturbed elliptic problems with superlinear or asymptotically linear nonlinearities, Calc. Var. Partial Differential Equations, 21 (2004), 287-318. 1

[20] N. Laskin, Fractional quantum mechanics and Léry path integrals, Phys. Lett. A, 268 (2000), 298-305. 1

[21] N. Laskin, Fractional Schrödinger equation, Phys. Rev. E, 66 (2002), 7 pages. 1

[22] L. Li, V. Rădulescu, D. Repovš, Nonlocal Kirchhoff superlinear equations with indefinite nonlinearity and lack of compactness, Int. J. Nonlinear Sci. Numer. Simul., 17 (2016), 325-333. 1 
[23] G.-B. Li, A. Szulkin, An asymptotically periodic Schrödinger equation with indefinite linear part, Commun. Contemp. Math., 4 (2002), 763-776. 1

[24] H. F. Lins, E. A. B. Silva, Quasilinear asymptotically periodic elliptic equations with critical growth, Nonlinear Anal., 71 (2009), 2890-2905. 1, 1.3, 2.5

[25] P. H. Rabinowitz, Minimax methods in critical point theory with applications to differential equations, CBMS Regional Conference Series in Mathematics, Published for the Conference Board of the Mathematical Sciences, Washington, DC; by the American Mathematical Society, Providence, RI, (1986). 1

[26] P. H. Rabinowitz, On a class of nonlinear Schrödinger equations, Z. Angew. Math. Phys., 43 (1992), 270-291. 1

[27] M. Schechter, A variation of the mountain pass lemma and applications, J. London Math. Soc., 44 (1991), 491-502. 2.4

[28] S. Secchi, Ground state solutions for nonlinear fractional Schrdinger equations in $\mathbb{R}^{N}$, J. Math. Phys., 54 (2013), 17 pages. $1,1,2.2$

[29] S. Secchi, On fractional Schrödinger equations in $\mathbb{R}^{N}$ without the Ambrosetti-Rabinowitz condition, Topol. Methods Nonlinear Anal., 47 (2016), 19-41. 1, 2.1

[30] X.-D. Shang, J.-H. Zhang, Ground states for fractional Schrödinger equations with critical growth, Nonlinearity, 27 (2014), 187-207. 1

[31] E. A. B. Silva, G. F. Vieira, Quasilinear asymptotically periodic Schrödinger equations with critical growth, Calc. Var. Partial Differential Equations, 39 (2010), 1-33. 1, 2.10, 2

[32] E. A. B. Silva, G. F. Vieira, Quasilinear asymptotically periodic Schrödinger equations with subcritical growth, Nonlinear Anal., 72 (2010), 2935-2949. 1

[33] A. Szulkin, T. Weth, The method of Nehari manifold, Handbook of nonconvex analysis and applications, Int. Press, Somerville, MA, (2010), 597-632. 1.3

[34] J.-G. Tan, The Brezis-Nirenberg type problem involving the square root of the Laplacian, Calc. Var. Partial Differential Equations, 42 (2011), 21-41. 1

[35] K.-M. Teng, Multiple solutions for a class of fractional Schrödinger equations in $\mathbb{R}^{\mathbf{N}}$, Nonlinear Anal. Real World Appl., 21 (2015), 76-86. 1

[36] H. Weitzner, G. M. Zaslavsky, Some applications of fractional equations, Chaotic transport and complexity in classical and quantum dynamics, Commun. Nonlinear Sci. Numer. Simul., 8 (2003), 273-281. 1

[37] M. Willem, Minimax theorems, Progress in Nonlinear Differential Equations and their Applications, Birkhäuser Boston, Inc., Boston, MA, (1996). 1

[38] H. Zhang, J.-X. Xu, F.-B. Zhang, Existence and multiplicity of solutions for superlinear fractional Schrödinger equations in $\mathbb{R}^{\mathrm{N}}$, J. Math. Phys., 56 (2015), 13 pages. 1, 1, 1.3, 2.10

[39] X. Zhang, B.-L. Zhang, D. Repovš, Existence and symmetry of solutions for critical fractional Schrödinger equations with bounded potentials, Nonlinear Anal., 142 (2016), 48-68.

[40] X. Zhang, B.-L. Zhang, M.-Q. Xiang, Ground states for fractional Schrödinger equations involving a critical nonlinearity, Adv. Nonlinear Anal., 5 (2016), 293-314. 1 